\title{
Addressing Cognitive Challenges in Schizophrenia: A Public Health Perspective
}

\author{
Jagadisha Thirthalli ${ }^{1}$
}

Received: 27 November 2015/ Accepted: 28 November 2015/Published online: 7 December 2015

(C) Springer India Pvt. Ltd. 2015

Keywords Schizophrenia $\cdot$ Cognitive deficit $\cdot$ Public health

\section{Cognitive Deficits and Disability in Schizophrenia}

Deficits in cognitive functions are ubiquitous in schizophrenia. Nearly all patients with this disorder experience deficits in neurocognitive domains including attention, memory, speed of processing, reasoning and problem solving and social cognitive domains including theory of mind, emotion recognition, social cue recognition, etc. [1]. These deficits precede the onset of first psychotic episode [2] and persist through episodes of psychotic exacerbation [3] and relative quiescence [4]. Importantly, they are consistently associated with patients' social and occupational functioning-nearly $20-60 \%$ of the variance in their functioning is explained by their performance on tests of cognitive functions [5]. Schizophrenia causes substantial disability-indeed it accounts for $0.5 \%$ of all-cause disability adjusted life years (DALY) [6]. Given that cognitive deficits associated with schizophrenia contribute considerably to disability, public health significance of addressing the challenge of cognitive deficits of schizophrenia cannot be overemphasized.

Jagadisha Thirthalli

jagatth@yahoo.com

1 Department of Psychiatry, National Institute of Mental Health and Neurosciences (NIMHANS), Bengaluru, India

\section{Cognitive Training in Schizophrenia}

A number of treatment approaches have shown promise in improving cognitive functions in schizophrenia. Several terms have been used interchangeably to describe these approaches-these include cognitive remediation, cognitive enhancement training, cognitive training, cognitive rehabilitation, etc. Most of these aim to effect learning and adaptive plastic changes in neural systems using specifically defined and controlled learning exercises [7]. Metaanalyses have shown that these approaches are effective in improving cognitive abilities of patients with schizophrenia with small to moderate effect sizes [8, 9]. Improvements have been noted not just in cognitive functions that the training methods targeted, but also in cognitive domains that were not directly targeted as well as measures of global cognitive abilities. Not surprisingly, the response to cognitive training response is associated with structural and functional changes in prefrontal area of the brain $[10,11]$. The gains of cognitive training are likely to be durable for several months beyond the training period [12, 13]. The gains of cognitive training interventions go beyond mere improvement in performance in cognitive tests-patients show improvement in measures of everyday functioning also. What's more, improvement in global functioning were indeed attributable to improvement in cognitive performance [14].

\section{Cognitive Training in Schizophrenia: Public Health Perspective}

Targeting cognitive deficits of schizophrenia forms an important aspect of management of schizophrenia, specifically in order to reduce the disability due to the disorder. 
However, there are a number of challenges in making the gains of cognitive training available to most of those who need them. Nearly all studies have been conducted in academic settings using highly trained staff as part of research initiatives. Their utility is yet to be tested in the real-world situation in places where there is no access to academic settings and trained manpower. Attempts of delivering cognitive training in public health systems using less trained staff have highlighted a number of problems at the implementation level-these included lack of protected time to deliver the intervention, lack of resources like therapy rooms, equipment, etc., poor funding and lack of organizational support [15].

In this context, it is useful to note a few attempts to develop low-cost alternatives to "conventional" cognitive training. There is evidence that internet-based targeted cognitive training targeting neurocognition and social cognition is feasible and useful in improving cognitive performance of individuals at clinically high risk to develop psychosis [16]. There is preliminary evidence that such training is useful in improving cognitive abilities of elderly individuals with mild cognitive impairment [17]. Research exploring such interventions are lacking in the field of cognitive rehabilitation of schizophrenia. These are relatively low-cost interventions, which have higher likelihood of acceptability and adherence. These qualities of such interventions make them promising strategies to be explored as cognitive training tools in schizophrenia.

Majority of patients with schizophrenia live in the developing world, with traditional family systems. Family caregivers have been the de-facto case managers in many cultures. There is evidence that low-cost, home-based cognitive training, in which family members actively supervised patients' engagement with training tasks with minimal assistance from professionals, improved performance of schizophrenia patients on a range of cognitive tasks [18]. If replicated, such strategies can be potential game-changers in providing cognitive training for individuals with schizophrenia in low-resource settings. Cognitive adaptation training aims to bypass the cognitive deficits that adversely affect patients' everyday functioning [19]. Research is under way to explore if such interventions, delivered by nurses, could be cost-effective strategies to improve functionality of patients [20]. Such strategies could become additional public health tools to address the challenge cognitive disability of schizophrenia.

An interesting finding of the meta-analyses of cognitive training in schizophrenia was that the interventions generally worked better if they were combined with other measures of psychiatric rehabilitation like vocational training and/or supported employment services [8, 13, 21]. This suggests an interesting interaction between psychiatric rehabilitative interventions and cognitive training. The exact nature of such an interaction remains to be explored. However, it is conceivable that cognitive training works better when the intervention happens in conjunction with active exposure to situations where cognitive abilities are put to practice in real-life situations. It also posits an interesting possibility that exposure to real-life challenges, within the context of therapeutic engagement by professionals, may lead to amelioration of cognitive deficits. Results of a randomized controlled trial of cognitive adaptive training by Fredrick and colleagues [22] lends some support to this. Their mediational analysis showed that improvement in real-world functioning resulted in improvement in performance in cognitive tasks, suggesting that attaining functional outcomes can improve cognitive functioning. This opens up another alternative to "conventional" cognitive training-providing psychiatric rehabilitation interventions targeting patients' everyday functioning. The latter may, in turn, result in better cognitive functioning. At this juncture, this proposition is speculative, requiring validation by empirical evidence. Public health strategies for improving the outcome of major psychiatric illnesses naturally include comprehensive, community-based rehabilitation interventions. Such interventions can potentially double up as alternative strategies to improve cognitive abilities of patients. Research exploring the role of different rehabilitative efforts in possibly ameliorating cognitive deficits of schizophrenia is of immense public health importance and need attention of researchers and funding agencies alike.

\section{References}

1. Nuechterlein KH, Barch DM, Gold JM, Goldberg TE, Green MF, Heaton RK. Identification of separable cognitive factors in schizophrenia. Schizophr Res. 2004;72(1):29-39.

2. Lee TY, Hong SB, Shin NY, Kwon JS. Social cognitive functioning in prodromal psychosis: a meta-analysis. Schizophr Res. 2015;164(1-3):28-34.

3. Green MF, Bearden CE, Cannon TD, Fiske AP, Hellemann GS, Horan WP, et al. Social cognition in schizophrenia, part 1: performance across phase of illness. Schizophr Bull. 2012;38(4):854-64.

4. Mehta UM, Thirthalli J, Naveen Kumar C, Keshav Kumar J, Keshavan MS, Gangadhar BN. Schizophrenia patients experience substantial social cognition deficits across multiple domains in remission. Asian J Psychiatr. 2013;6(4):324-9.

5. Green MF. What are the functional consequences of neurocognitive deficits in schizophrenia? Am J Psychiatry. 1996;153(3):321-30.

6. Whiteford HA, Ferrari AJ, Degenhardt L, Feigin V, Vos T. The global burden of mental, neurological and substance use disorders: an analysis from the global burden of disease study 2010. PLoS One. 2015;10(2):e0116820.

7. Keshavan MS, Vinogradov S, Rumsey J, Sherrill J, Wagner A. Cognitive training in mental disorders: update and future directions. Am J Psychiatry. 2014;171(5):510-22. 
8. Wykes T, Huddy V, Cellard C, McGurk SR, Czobor P. A metaanalysis of cognitive remediation for schizophrenia: methodology and effect sizes. Am J Psychiatry. 2011;168(5):472-85.

9. McGurk SR, Twamley EW, Sitzer DI, McHugo GJ, Mueser KT. A meta-analysis of cognitive remediation in schizophrenia. Am J Psychiatry. 2007;164(12):1791-802.

10. Eack SM, Hogarty GE, Cho RY, Prasad KM, Greenwald DP, Hogarty SS, et al. Neuroprotective effects of cognitive enhancement therapy against gray matter loss in early schizophrenia: results from a 2-year randomized controlled trial. Arch Gen Psychiatry. 2010;67(7):674-82.

11. Subramaniam K, Luks TL, Fisher M, Simpson GV, Nagarajan S, Vinogradov $\mathrm{S}$. Computerized cognitive training restores neural activity within the reality monitoring network in schizophrenia. Neuron. 2012;73(4):842-53.

12. Eack SM, Greenwald DP, Hogarty SS, Keshavan MS. One-year durability of the effects of cognitive enhancement therapy on functional outcome in early schizophrenia. Schizophr Res. 2010;120(1):210-6.

13. Wykes T, Reeder C, Williams C, Corner J, Rice C, Everitt B. Are the effects of cognitive remediation therapy (CRT) durable? Results from an exploratory trial in schizophrenia. Schizophr Res. 2003;61(2):163-74.

14. Eack SM, Pogue-Geile MF, Greenwald DP, Hogarty SS, Keshavan MS. Mechanisms of functional improvement in a 2-year trial of cognitive enhancement therapy for early schizophrenia. Psychol Med. 2011;41(6):1253-61.

15. Cairns A, Dark F, Batts M. Implementing cognitive remediation therapy: lessons from two public mental health services. Australas Psychiatry. 2013;21(5):476-80.
16. Hooker CI, Carol EE, Eisenstein T, Yin H, Lincoln SH, Tully LM, et al. A pilot study of cognitive training in clinical high risk for psychosis: initial evidence of cognitive benefit. Schizophr Res. 2014;157:314.

17. Finn M, McDonald S. Computerised cognitive training for older persons with mild cognitive impairment: a pilot study using a randomised controlled trial design. Brain Impair. 2011;12(03):187-99.

18. Hegde S, Rao SL, Raguram A, Gangadhar BN. Addition of home-based cognitive retraining to treatment as usual in first episode schizophrenia patients: a randomized controlled study. Indian J Psychiatry. 2012;54(1):15.

19. Velligan DI, Bow-Thomas CC, Huntzinger C, Ritch J, Ledbetter N, Prihoda TJ, et al. Randomized controlled trial of the use of compensatory strategies to enhance adaptive functioning in outpatients with schizophrenia. Am J Psychiatry. 2000;157(8):1317-28.

20. Stiekema AP, Quee PJ, Dethmers M, van den Heuvel ER, Redmeijer JE, Rietberg K, et al. Effectiveness and cost-effectiveness of cognitive adaptation training as a nursing intervention in longterm residential patients with severe mental illness: study protocol for a randomized controlled trial. Trials. 2015;16(1):49.

21. National Collaborating Centre for Mental Health: Schizophrenia. Core interventions in the treatment and management of schizophrenia in primary and secondary care. London: National Institute for Health and Clinical Excellence; 2009.

22. Fredrick MM, Mintz J, Roberts DL, Maples NJ, Sarkar S, Li X, et al. Is cognitive adaptation training (CAT) compensatory, restorative, or both? Schizophr Res. 2015;166(1):290-6. 